\title{
A Beneficial Role of Computer-aided Diagnosis System for Less Experienced Physicians in the Diagnosis of Thyroid Nodule on Ultrasound
}

\section{Sunyoung Kang}

Department of Internal Medicine, Seoul National University College of Medicine, Seoul

\section{Eunjung Lee}

School of Mathematics and Computing, Yonsei University, Seoul

\section{Chae Won Chung}

Department of Internal Medicine, Seoul National University College of Medicine, Seoul

\section{Han Na Jang}

Department of Internal Medicine, Seoul National University College of Medicine, Seoul

Joon Ho Moon

Department of Internal Medicine, Seoul National University Bundang Hospital, Seongnam

Yujin Shin

Department of Internal Medicine, Seoul National University Bundang Hospital, Seongnam

Kyuho Kim

Department of Internal Medicine, Seoul National University Bundang Hospital, Seongnam

\section{Ying Li}

Department of Internal Medicine, Seoul National University College of Medicine, Seoul

\section{Soo Myoung Shin}

Department of Internal Medicine, Seoul National University College of Medicine, Seoul

\section{Yoo Hyung Kim}

Department of Internal Medicine, Seoul National University Hospital, Seoul

\section{Seul Ki Kwon}

Department of Internal Medicine, Seoul National University College of Medicine, Seoul

\section{Chang Ho Ahn}

Department of Internal Medicine, Seoul National University Bundang Hospital, Seongnam

\section{Kyong Yeun Jung}

Department of Internal Medicine, Nowon Eulji Medical Center, Eulji University School of Medicine, Seoul

\section{A Ram Hong}

Department of Internal Medicine, Chonnam National University Medical School, Gwangju

\section{Young Joo Park}

Department of Internal Medicine, Seoul National University College of Medicine, Seoul

\section{Do Joon Park}

Department of Internal Medicine, Seoul National University College of Medicine, Seoul

\section{Jin Young Kwak}

Department of Radiology, Severance Hospital, Research Institute of Radiological Science, Yonsei University College of Medicine, Seoul

Sun Wook Cho ( $\nabla$ swchomd@gmail.com )

Department of Internal Medicine, Seoul National University College of Medicine, Seoul

\section{Research Article}

Keywords: thyroid nodule, thyroid cancer, ultrasonography, computer-aided diagnosis, deep learning 
DOI: https://doi.org/10.21203/rs.3.rs-568240/v1

License: (a) (1) This work is licensed under a Creative Commons Attribution 4.0 International License. Read Full License 


\section{Abstract}

Ultrasonography is the primary diagnostic tool for thyroid nodules, while the accuracy is highly operator-dependent. The aim of this study was to investigate whether ultrasonography with computer-aided diagnosis (CAD) has assisting roles to physicians in the diagnosis of thyroid nodules. 451 thyroid nodules $\left({ }^{3} 1 \mathrm{~cm}\right)$ evaluated by fine-needle aspiration cytology following surgery were included. $300(66.5 \%)$ of them were diagnosed as malignancy. Thirteen physicians who had 0 months $(E 0, n=8), 1$ year $(E 1, n=2)$, or more than 5 years $(E 5, n=3)$ of experience in ultrasonography reviewed the prepared ultrasound images of thyroid nodules before and after CAD assistance. The diagnostic performance of CAD was comparable to that of the E5 group, and better than those of the E0 and E1 groups. The AUC of the CAD for conventional PTC was higher than that for FTC and follicular variant PTC (0.925 vs. 0.499), independent of tumor size. CAD assistance significantly improved diagnostic performance in E0 group, but not in the E1 and E5 groups. In conclusion, the CAD system showed good performance in the diagnosis of conventional PTC. CAD assistance improved the diagnostic performance of physicians with less experience in ultrasonography, especially in the diagnosis of conventional PTC.

\section{Introduction}

Ultrasonography (US) is the primary diagnostic tool used to access the malignancy risk of thyroid nodules. However, the accuracy of diagnostic US is highly operator-dependent and requires years of experience and training to read US image ${ }^{1}$. To optimize the use of diagnostic US for assessing the malignancy risk of thyroid nodules, various US risk stratification systems have been developed by several societies, such as the American College of Radiology (ACR) ${ }^{2}$, the American Thyroid Association ${ }^{3}$, the European Thyroid Association ${ }^{4}$, the Korean Society of Thyroid Radiology ${ }^{5}$, and the American Association of Clinical Endocrinologists, American College of Endocrinology, and Associazione Medici Endocrinologi ${ }^{6}$. Nonetheless, these US risk stratification systems have shown differences in diagnostic performance depending on the study group ${ }^{7-9}$, and inter-observer variability for thyroid US can be high even when a single risk stratification system is used ${ }^{10}$.

Computer-Aided Diagnosis (CAD) systems have been developed and applied for US diagnostics in various medical fields, catching up with the rapidly developing techniques of machine learning. Several recent studies showed that the diagnostic performance of machine learning in US CAD systems was comparable to that of expert radiologists ${ }^{11-15}$. However, a meta-analysis including 4 studies from the Samsung CAD system and 1 study from independently developed CAD system from China demonstrated that the specificity and the diagnostic odds ratio of the CAD system were lower than those of the experienced radiologist, while the sensitivity of the CAD system was similar ${ }^{16}$. We recently developed another US CAD system for thyroid nodule diagnosis using a machine learning method involving a deep convolutional neural network (CNN) model ${ }^{17}$. This system showed comparable or higher diagnostic performance than that of expert radiologists, however further validation of its diagnostic performance in various clinical settings and exploration of appropriate clinical use is needed.

Thyroid nodules are a common medical problem, and US is widely employed in the diagnosis of thyroid nodules not only by expert radiologists in the hospital but also by physicians in the primary clinics. However, weather the US CAD system is beneficial to the less experienced physicians or in the primary care setting has not been fully studied yet. The aim of this study was to investigate the potential benefits of the US CAD system in the diagnosis of thyroid nodules for less experienced physicians.

\section{Results}

\section{Clinical characteristics of thyroid nodules}

The clinical characteristics of the thyroid nodules are presented in Table 1. Of the 451 enrolled thyroid nodules, 300 nodules (66.5\%) were surgically confirmed as malignant. Compared to the benign nodules, the malignant nodules were more frequently found in male patients $(29.3 \%$ vs. $15.2 \%, p=0.001)$ and were smaller on average $(1.81 \pm 1.0$ vs. $2.52 \pm 1.2 \mathrm{~cm}, p<0.001)$. Patients' mean age at the time of diagnosis was similar between groups. The cases of thyroid cancer were categorized as conventional papillary thyroid carcinoma (CPTC), follicular variant papillary thyroid carcinoma (fVPTC), follicular thyroid carcinoma (FTC), medullary thyroid carcinoma, poorly differentiated thyroid carcinoma, and anaplastic thyroid carcinoma. CPTC, fvPTC, and FTC accounted for $83.7 \%$, $7.0 \%$, and $7.3 \%$ of the malignant nodules, respectively. The tumor size was $<2 \mathrm{~cm}$ in $78.9 \%$ of cPTCs, while $65.1 \%$ of FTCs and fvPTCs combined (FTC/fvPTC) had a size of $\geq 2 \mathrm{~cm}(p<0.001$, Table S1). Of the benign nodules, $38.4 \%$ were follicular adenoma, $31.8 \%$ were nodular hyperplasia, $23.8 \%$ were NIFTP, and $6.0 \%$ were other benign lesions. 
Table 1

Baseline Characteristics of Thyroid Nodules

\begin{tabular}{|c|c|c|c|c|}
\hline & Total & Benign & Malignancy & $P$-value \\
\hline $\mathrm{N}(\%)$ & 451 & $151(33.5)$ & $300(66.5)$ & \\
\hline Age of diagnosis, yrs & $50.0 \pm 14.3$ & $51.7 \pm 13.5$ & $49.1 \pm 14.6$ & 0.064 \\
\hline Male sex, n (\%) & $112(24.8)$ & $23(15.2)$ & $89(29.3)$ & 0.001 \\
\hline Size, $\mathrm{cm}$ & $2.05 \pm 1.1$ & $2.52 \pm 1.2$ & $1.81 \pm 1.0$ & $<0.001$ \\
\hline \multicolumn{5}{|c|}{ Histologic subtype, n (\%) } \\
\hline сPTC & - & - & $251(83.7)$ & \\
\hline fvPTC & - & - & $21(7.0)$ & \\
\hline FTC & - & - & $22(7.3)$ & \\
\hline MTC/PDTC/ATC & - & - & $6(2.0)$ & \\
\hline Follicular adenoma & - & $58(38.4)$ & - & \\
\hline Nodular hyperplasia & - & $48(31.8)$ & - & \\
\hline NIFTP & - & $36(23.8)$ & - & \\
\hline Other benign lesions & - & $9(6.0)$ & - & \\
\hline \multicolumn{5}{|c|}{$\begin{array}{l}\text { cPTC, conventional papillary thyroid carcinoma; fvPTC, follicular variant papillary thyroid carcinoma; FTC, follicular thyroid } \\
\text { carcinoma; MTC, medullary thyroid carcinoma; PDTC, poorly differentiated thyroid carcinoma; ATC, anaplastic thyroid carcinoma; } \\
\text { NIFTP, noninvasive follicular thyroid neoplasm with papillary-like nuclear features, } p \text {-value for benign vs. malignancy }\end{array}$} \\
\hline
\end{tabular}

\section{Diagnostic performance of thyroid US CAD}

The diagnostic performance of the CAD system is presented in Table 2 and Fig. 2. Overall, the AUC was 0.855 (Fig. $2 A$ ), and the sensitivity, specificity, PPV, and NPV, and accuracy were $85.3 \%, 63.6 \%, 82.3 \%, 68.6 \%$, and $78.0 \%$, respectively (Table 2). In the subgroup analysis, the CAD system showed higher diagnostic performance for thyroid nodules with a size $<2 \mathrm{~cm}$ than for larger nodules ( $\geq 2$ $\mathrm{cm}$ ) in terms of AUC ( 0.895 vs. 0.751 , Fig. 2 B and 2 C), sensitivity ( $94.4 \%$ vs. $62.4 \%)$, PPV ( $84.9 \%$ vs. $73.6 \%)$, NPV (70.7\% vs. $67.6 \%)$, and accuracy ( $82.9 \%$ vs. $70.2 \%)$. Since cPTC was significantly smaller than the other cancers (Table S1), we then analyzed the diagnostic performance of the CAD system according to histologic subgroup. Compared to FTC/fvPTC, a higher AUC was found for CPTC (0.925 vs. 0.499 , Fig. $2 \mathrm{D}$ and $2 \mathrm{E}$ ). For CPTC, the CAD system also showed higher sensitivity ( $94.4 \%$ vs. $34.9 \%)$, PPV ( $85.3 \%$ vs. $26.8 \%)$, NPV ( $84.1 \%$ vs. $72.5 \%)$, and accuracy ( $85.0 \%$ vs. $56.3 \%$ ). Interestingly, within the cPTC group, the diagnostic performance of the CAD system was similar regardless of size (AUC, 0.919 for nodules $<2 \mathrm{~cm}$, Fig. $3 \mathrm{~A} ; 0.907$ for nodules $\geq 2 \mathrm{~cm}$, Fig. 3B). 
Table 2

Diagnostic Performance of Computer-Aided Diagnosis (CAD)

\begin{tabular}{|c|c|c|c|c|c|c|}
\hline & AUC & Sensitivity (\%) & Specificity (\%) & PPV (\%) & NPV (\%) & Accuracy (\%) \\
\hline \multirow[t]{2}{*}{ Total } & 0.855 & 85.3 & 63.6 & 82.3 & 68.6 & 78.0 \\
\hline & $(0.820-0.889)$ & $(0.822-0.881)$ & (57. 4-69.1) & $(79.3-85.0)$ & $(61.9-74.5)$ & $(73.9-81.7)$ \\
\hline \multicolumn{7}{|l|}{ Size group } \\
\hline \multirow[t]{2}{*}{ Size $<2 \mathrm{~cm}$} & 0.895 & 94.4 & 44.6 & 84.9 & 70.7 & 82.9 \\
\hline & $(0.857-0.932)$ & $(91.6-96.7)$ & $(35.4-52.0)$ & $(82.4-87.0)$ & $(56.1-82.5)$ & $(78.6-86.3)$ \\
\hline \multirow[t]{2}{*}{ Size $\geq 2 \mathrm{~cm}$} & 0.751 & 62.4 & 77.9 & 73.6 & 67.7 & 70.2 \\
\hline & $(0.678-0.825)$ & $(54.6-69.0)$ & $(70.2-84.5)$ & $(64.4-81.5)$ & $(61.0-73.4)$ & $(62.5-76.8)$ \\
\hline \multicolumn{7}{|l|}{ Histologic subtypes } \\
\hline \multirow[t]{2}{*}{ cPTC vs. benign } & 0.925 & 94.4 & 64.3 & 85.3 & 84.1 & 85.0 \\
\hline & $(0.899-0.952)$ & $(91.6-96.6)$ & $(58.1-69.0)$ & $(82.7-87.2)$ & $(76.0-90.2)$ & $(81.1-87.9)$ \\
\hline \multirow[t]{2}{*}{ FTC and fvPTC vs. benign } & 0.499 & 34.9 & 64.3 & 26.8 & 72.5 & 56.3 \\
\hline & $(0.399-0.599)$ & $(21.0-50.9)$ & $(54.9-73.1)$ & $(15.8-40.3)$ & $(62.8-80.9)$ & $(49.7-63.7)$ \\
\hline
\end{tabular}

\section{Diagnostic performance of physicians before and after CAD assistance}

Next, the diagnostic performance was compared between the CAD system and physicians with various levels of experience (the E0, E1, and E5 groups) (Table 3). The E0 group showed significantly lower sensitivity $(78.9 \%$ vs. $85.3 \%, p=0.003)$, Specificity $(45.0 \%$ vs. $63.6 \%$, $p<0.001)$, PPV ( $75.2 \%$ vs. $82.3 \%, p=0.002)$, NPV ( $49.5 \%$ vs. $68.6 \%, p<0.001)$, and accuracy $(67.5 \%$ vs. $78.0 \%, p<0.001)$ than the CAD system. The E1 group showed significantly lower sensitivity $(80.3 \%$ vs. $85.3 \%, p=0.015)$ and accuracy $(74.6 \%$ vs. $78.0 \%, p=0.050)$ than the CAD system. The E5 group showed higher sensitivity compared to that of the CAD system $(89.4 \%$ vs. $85.3 \%, p=0.017)$. CAD assistance significantly improved the diagnostic performance of the E0 group. The sensitivity and accuracy significantly improved after CAD assistance (sensitivity, 78.9\% [before] vs. 85.3\% [after], $p=0.002$; accuracy, 67.5\% [before] vs. 73.9\% [after], $p=0.001$, Table 3). Meanwhile, the diagnostic performance of the E1 and E5 groups did not significantly change after CAD assistance (Table 3). 
Table 3

Diagnostic Performance of Physicians with Different Levels of Experience Before and After CAD Assistance

\begin{tabular}{|c|c|c|c|c|c|c|c|c|c|c|c|c|c|}
\hline & \multirow[t]{2}{*}{ CAD } & \multicolumn{4}{|l|}{ EO } & \multicolumn{4}{|l|}{ E1 } & \multicolumn{4}{|l|}{ E5 } \\
\hline & & Before & After & $P^{a}$ & $P^{b}$ & Before & After & $P^{a}$ & $P^{b}$ & Before & After & $P^{a}$ & $P^{b}$ \\
\hline Sensitivity & 85.3 & $78.9 \%$ & $85.3 \%$ & 0.003 & 0.002 & $80.3 \%$ & $81.3 \%$ & 0.015 & 0.356 & $89.4 \%$ & $90.4 \%$ & 0.017 & 0.292 \\
\hline Specificity & 63.6 & $45.0 \%$ & $51.2 \%$ & $\begin{array}{l}< \\
0.001\end{array}$ & 0.076 & $63.2 \%$ & $64.2 \%$ & 0.498 & 0.400 & $57.6 \%$ & $58.3 \%$ & 0.079 & 0.463 \\
\hline PPV & 82.3 & $75.2 \%$ & $78.7 \%$ & 0.002 & - & $82.2 \%$ & $82.8 \%$ & 0.515 & - & $80.7 \%$ & $81.1 \%$ & 0.261 & - \\
\hline NPV & 68.6 & $49.5 \%$ & $66.5 \%$ & $\begin{array}{l}< \\
0.001\end{array}$ & - & $62.2 \%$ & $63.5 \%$ & 0.070 & - & $73.1 \%$ & $75.2 \%$ & 0.133 & - \\
\hline Accuracy & 78.0 & $67.5 \%$ & $73.9 \%$ & $\begin{array}{l}< \\
0.001\end{array}$ & 0.001 & $74.6 \%$ & $75.6 \%$ & 0.050 & 0.310 & $77.4 \%$ & $79.6 \%$ & 0.396 & 0.134 \\
\hline \multicolumn{14}{|c|}{$\begin{array}{l}\text { ACR-TIRADS } 4 \text { was used as the cut-off to calculate the diagnostic performance of physicians. CAD, computer-aided diagnosis; } \\
\text { E0/E1/E5, physicians with } 0 \text { month/ } 1 \text { year/ } 5 \text { years of experience; Before, physicians before CAD assistance; After, physicians after } \\
\text { CAD assistance. PPV, positive preeedictive value; NPV, negative predictive value. }\end{array}$} \\
\hline
\end{tabular}

A subgroup analysis was performed according to the subtype of thyroid cancers. The AUC of the physicians was higher for PTC than for FTC/fvPTC (0.737-0.902 vs. 0.437-0.605), and CAD assistance significantly improved the AUC in most of the E0 group and a subset of experienced physicians (E1 and E5 groups) for the diagnosis of CPTC, but not for FTC/fvPTC (Table S2). With CAD assistance, the mean sensitivity and accuracy for diagnosing cPTC significantly improved in the E0 group (sensitivity, 83.4\% [before] vs. $91.6 \%$ [after], $p<0.001$; accuracy, $71.3 \%$ [before] vs. 79.4\% [after], $p<0.001$ ), but not in the E1 and E5 groups (Table 4). Additionally, the mean accuracy for diagnosing CPTC significantly increased after CAD assistance, regardless of nodule size (nodules $<2 \mathrm{~cm}, 74.3 \%$ [before] vs. 81.5\% [after], $p=0.003$; nodules $\geq 2 \mathrm{~cm}, 64.7 \%$ [before] vs. $74.6 \%$ [after], $p=0.014$ ) in the E0 group (Table S3). 
Table 4

Comparisons of Diagnostic Performances Between CAD and Physicians Before and After CAD Assistance According to the Pathologic Subtype

\begin{tabular}{|c|c|c|c|c|c|c|c|c|c|c|c|c|c|}
\hline & \multirow[t]{2}{*}{ CAD } & \multicolumn{4}{|l|}{ EO } & \multicolumn{4}{|l|}{ E1 } & \multicolumn{4}{|l|}{ E5 } \\
\hline & & Before & After & $P^{a}$ & $P^{b}$ & Before & After & $P^{a}$ & $P^{b}$ & Before & After & $P^{a}$ & $P^{b}$ \\
\hline \multicolumn{14}{|l|}{ cPTC } \\
\hline Sensitivity & 94.4 & $83.4 \%$ & $91.6 \%$ & $\begin{array}{l}< \\
0.001\end{array}$ & $\begin{array}{l}< \\
0.001\end{array}$ & $87.8 \%$ & $88.8 \%$ & $\begin{array}{l}< \\
0.001\end{array}$ & 0.309 & $94.6 \%$ & $95.5 \%$ & 0.564 & 0.243 \\
\hline Specificity & 64.3 & $45.0 \%$ & $52.7 \%$ & $\begin{array}{l}< \\
0.001\end{array}$ & 0.060 & $65.7 \%$ & $67.0 \%$ & 0.384 & 0.452 & 60.1 & 61.7 & 0.202 & 0.388 \\
\hline PPV & 85.3 & $78.0 \%$ & $81.8 \%$ & 0.002 & - & $85.6 \%$ & $86.2 \%$ & 0.460 & - & 83.7 & 84.5 & 0.272 & - \\
\hline NPV & 84.1 & $53.2 \%$ & $77.8 \%$ & $\begin{array}{l}<.001 \\
0.0\end{array}$ & - & $71.9 \%$ & $73.6 \%$ & 0.006 & - & 83.1 & 86.5 & 0.470 & - \\
\hline Accuracy & 85.0 & $71.3 \%$ & $79.4 \%$ & $\begin{array}{l}< \\
0.001\end{array}$ & $\begin{array}{l}< \\
0.001\end{array}$ & $80.9 \%$ & $82.0 \%$ & 0.019 & 0.307 & 83.6 & 84.9 & 0.265 & 0.265 \\
\hline \multicolumn{14}{|c|}{ FTC and fvPTC } \\
\hline Sensitivity & 34.9 & $53.8 \%$ & $50.6 \%$ & 0.010 & 0.432 & $38.4 \%$ & $39.5 \%$ & 0.380 & 0.497 & $61.2 \%$ & $62.0 \%$ & $\begin{array}{l}< \\
0.001\end{array}$ & 0.540 \\
\hline Specificity & 64.3 & $45.0 \%$ & $52.7 \%$ & $\begin{array}{l}< \\
0.001\end{array}$ & 0.060 & $65.7 \%$ & $67.0 \%$ & 0.414 & 0.452 & $56.5 \%$ & $57.4 \%$ & 0.053 & 0.460 \\
\hline PPV & 26.8 & $25.3 \%$ & $27.6 \%$ & 0.449 & - & $30.4 \%$ & $33.5 \%$ & 0.335 & & $34.2 \%$ & $35.5 \%$ & 0.152 & - \\
\hline NPV & 72.5 & $73.3 \%$ & $76.3 \%$ & 0.546 & - & $74.1 \%$ & $74.7 \%$ & 0.397 & & $79.2 \%$ & $80.4 \%$ & 0.066 & - \\
\hline Accuracy & 56.3 & $47.4 \%$ & $52.1 \%$ & 0.015 & 0.139 & $58.2 \%$ & $59.5 \%$ & 0.345 & 0.402 & $57.6 \%$ & $58.2 \%$ & 0.403 & 0.469 \\
\hline \multicolumn{14}{|c|}{ ACR-TIRADS 4 was used as the cut-off to calculate the diagnostic performance of physicians. } \\
\hline \multicolumn{14}{|c|}{$\begin{array}{l}\text { CAD, computer-aided diagnosis; E0/E1/E5, physicians with } 0 \text { month/ } 1 \text { year/ } 5 \text { years of experience; Before, physicians before CAD } \\
\text { assistance; After, physicians after CAD assistance; PPV, positive predictive value; NPV, negative predictive value; } C P T C \text {, conventiona } \\
\text { papillary thyroid carcinoma; FTC, follicular thyroid carcinoma; fvPTC, follicular variant papillary thyroid carcinoma. } P^{a}, C A D \text { vs. } \\
\text { before; } P^{b} \text {, before vs. after. }\end{array}$} \\
\hline
\end{tabular}

\section{Discussion}

In this study, the diagnostic performance for assessing the malignancy risk of thyroid nodules using US was compared between the CAD system and physicians with various levels of US experience, and the role of CAD assistance for physicians was investigated. The AUC of the CAD system was 0.855 for all thyroid nodules and 0.925 for nodules diagnosed as CPTC, which was much higher than the AUC for nodules diagnosed as FTC/fvPTC. The diagnostic performance of physicians without US experience (E0 group) was significantly lower than that of the CAD system, and CAD assistance improved their performance. Collectively, the present study demonstrated the beneficial role of assistance from the US CAD system for physicians with insufficient US training.

US is the most sensitive and widely used diagnostic tool for thyroid nodule assessment. Malignant nodules (especially PTCs) have specific US features in terms of echogenicity, solidity, orientation, and the presence of microcalcification ${ }^{3,18}$. Nonetheless, the reported diagnostic value of US varies considerably across studies, with high inter-performer and inter-observer variability. Although several guidelines have been established by related societies ${ }^{2-6}$, high inter-observer variability was still observed even among board-certified radiologists $(K=0.51)^{10}$. In the real-world practice the diagnostic performance of US in accessing thyroid nodule showed big difference depending on the experience levels of operators. With this background, CAD systems have shown promise in overcoming these limitations of US performance, and robust technical developments have recently been achieved through breakthroughs in deep learning technology based on artificial neural networks $11,12,14,15$. Indeed, previous studies reported that CAD systems developed using deep learning models showed comparable diagnostic performance to expert radiologists, and the present study (AUC, 0.855) also showed similar performance to that reported in other studies (AUC, $0.87-0.947$ ) ${ }^{14,15,19}$. 
The present study demonstrated that a US CAD system established by a deep learning method (CNN) can furnish useful diagnostic assistance for less experienced physicians. Since US is widely used both by well-trained radiologists and by physicians in their general clinics, the present study stated the first step to verify the clinical use of the US CAD system. However, several points need to be considered regarding the application of the present CAD system in the practice of primary care physicians. In the development and validation process of the current CAD system, both the training set and the study set of nodules were enrolled from a tertiary referral hospital which are different from those of the primary care system. Furthermore, the enrolled nodules in the present study were all surgically diagnosed, which can lead to selection bias. Thus, further study is needed in primary care conditions.

Additionally, the present study has several limitations. First, the US CAD system was originally developed using nodules $1 \mathrm{~cm}$ or larger, so it cannot be applied to nodules smaller than $1 \mathrm{~cm}$. Although, the present study showed excellent results for the diagnosis of PTC using the CAD system in nodules of any size, an expanded CAD system would be needed for micro-nodules, which are identified at an increasing frequency. Second, the CAD system showed no beneficial role for the diagnosis of FTC/fvPTC. The AUC for FTC/fvPTC was 0.499 , which was similar to that of physicians regardless of experience. Unlike CPTC, the US characteristics of FTC/fvPTC are very heterogeneous and non-specific ${ }^{20-23}$, and play a minimal role in the preoperative diagnosis ${ }^{24}$. Additionally, the US CAD system used in the present study was trained using PTC-dominant learning materials, as $96.5 \%$ of the nodule were PTCs. A challenge for further research would be to develop a highly advanced CAD system using artificial intelligence with sufficient data on FTC/fvPTCs.

In conclusion, the CAD system showed good diagnostic performance and had a beneficial assistive role for physicians with less US experience in assessing the malignancy risk of thyroid nodules, especially in PTCs. Therefore, this US CAD system can be a beneficial tool to assess less-experienced physicians in PTC-dominant areas.

\section{Materials And Methods}

\section{Study population}

A total of 5,581 US images of thyroid nodules from 4,143 patients who had undergone fine-needle aspiration (FNA) at the Department of Endocrinology, Seoul National University Hospital from April 2014 to June 2019 were consecutively recruited and reviewed. The

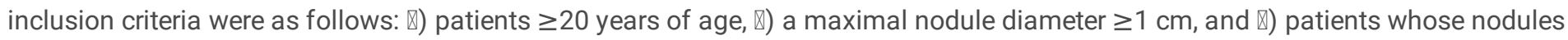
were pathologically confirmed by surgery. Finally, 451 thyroid nodules were enrolled (Figure 1). Thirteen physicians with various levels of US experience were recruited from three referral hospitals. Eight of them were third-year residents of internal medicine with basic thyroid imaging training (designated as the E0 group), 2 of them were endocrine fellows completing 1-year training course of thyroid US imaging and FNA procedures (designated as the E1 group), and 3 of them were endocrine faculties with more than 5 years of experience in thyroid USG imaging and FNA procedures (designated as the E5 group). This study was approved by the Institutional Review Board of Seoul National University Hospital (IRB No. 1911-039-1076). Written informed consent has been obtained from each patient after full explanation of the purpose and nature of all procedures used. All methods were carried out in accordance with relevant guidelines and regulations.

\section{Cytologic and histologic evaluation of thyroid nodules}

According to the recommendation of the Korean Thyroid Imaging Reporting and Data System (K-TIRADS) ${ }^{5}$, all nodules were evaluated by US and FNA was performed for suspicious nodules by experienced physicians. The cytology results were reported using the Bethesda System for Reporting Thyroid Cytopathology ${ }^{25}$ by an expert pathologist who had more than 10 years of experience at a tertiary hospital. Surgery was performed in patients with Bethesda cytology categories IV, V, and VI. Additionally, patients having nodules with Bethesda cytology categories II or III also underwent surgery if they have large nodule size, simultaneous presence of other nodules confirmed as malignancy, or the presence of compressive symptoms. Noninvasive follicular thyroid neoplasm with papillary-like nuclear features (NIFTP) was defined as a benign lesion.

\section{Ultrasonography examinations}

US examinations were performed using high-resolution ultrasound machines (LOGIQ7; GE Healthcare, Milwaukee, WI, USA or Affinity 50G; Philips Healthcare, Bothell, WA, USA). Each system was equipped with a linear, high-frequency transducer (5-14 MHz). After screening patients, we selected the most representative image in each thyroid nodule, and saved it as a JPEG file. A square region of interest for each nodule was drawn by an expert radiologist (J.Y.K). After the CAD system calculated the cancer probability, the US images of thyroid nodules were reviewed by 13 physicians. 
The physicians reviewed the US images in two stages using the ACR-TIRADS ${ }^{2}$. First, they scored US images without assistance from the CAD system, and then they re-scored the US images with CAD assistance. The CAD system showed results dichotomized as cancer (1) or benign (0). Based on this information, they re-reviewed the same US image again, and then continued to review the next US image. All physicians were blinded to the patients' clinical information and pathology results.

\section{US CAD system}

To evaluate malignancy risk, we used our CAD system that had been developed using a deep CNN model. The detailed development protocol of the US CAD system has been published previously ${ }^{17}$. Briefly, the algorithm was trained using 13,560 US images of thyroid nodules that were either surgically or cytologically proven as benign or malignant. For internal and external validation tests, surgically confirmed thyroid nodules were obtained from three tertiary hospitals and the tests verified that the diagnostic performance of the CAD system was comparable or higher than that of expert radiologists. Once a US image is input into the CAD system, the results are presented as cancer probabilities (\%), and the images are also classified as malignant or benign, with a cut-off value of a $50 \%$ probability of malignancy.

\section{Statistical analysis}

Data are described as the mean \pm standard deviation for continuous variables, and as numbers (percentages) for categorical variables. To compare mean values between the two groups, the Student $t$ test was used for continuous variables, and the chi-square test was used for categorical variables. To assess the diagnostic performance of the CAD system and physicians, receiver operating characteristic curves were constructed and the area under the curve (AUC) was calculated. The cut-off value of ACR-TIRADS was determined using the Youden index, and TR4 was used as the cut-off to calculate the sensitivity, specificity, positive predictive value (PPV), negative predictive value (NPV), and accuracy of physicians. To compare diagnostic performance between the CAD system and physicians, the mean accuracy, sensitivity, and specificity of physicians were compared to those of the CAD system using the binominal test. The statistical analysis was performed using STATA version 13.1 (StataCorp, College Station, TX, USA), and $p$-values $<0.05$ were considered to indicate statistical significance for all tests.

\section{Declarations}

\section{Author contributions}

All authors were involved in revising the manuscript and gave final approval. S.K., E.L., J.Y.K. and S.W.C. designed the study, analyzed the data. C.W.J., H.N.J., J.H.M., Y.S., K.K., Y.L., S.M.S., Y.H.K., S.K.K., C.H.A., K.Y.J., A.R.H., S.W.C. reviewed and scored the US image. S.K. wrote the manuscript, and Y.J.P., D.J.P., J.Y.K., S.W.C. critically reviewed the manuscript.

\section{Declaration of interest}

All authors declare that there is no conflict of interest that could be perceived as prejudicing the impartiality of the research reported

\section{Acknowledgments}

This work was supported by a National Research Foundation of Korea (NRF) grant funded by the Korean government (MSIT) (2019R1A2C1002375 and 2021R1A2C2007492). The funders had no role in study design, data collection and analysis, decision to publish, or preparation of the manuscript.

\section{Data Availability Statement}

The data presented in this study are available on request from the corresponding author.

\section{References}

1. Huang, Q., Zhang, F. \& Li, X. Machine Learning in Ultrasound Computer-Aided Diagnostic Systems: A Survey. Biomed Res Int 2018, 5137904, doi:10.1155/2018/5137904 (2018).

2. Tessler, F. N. et al. ACR Thyroid Imaging, Reporting and Data System (TI-RADS): White Paper of the ACR TI-RADS Committee. J Am Coll Radio/ 14, 587-595, doi:10.1016/j.jacr.2017.01.046 (2017). 
3. Haugen, B. R. et al. 2015 American Thyroid Association Management Guidelines for Adult Patients with Thyroid Nodules and Differentiated Thyroid Cancer: The American Thyroid Association Guidelines Task Force on Thyroid Nodules and Differentiated Thyroid Cancer. Thyroid 26, 1-133, doi:10.1089/thy.2015.0020 (2016).

4. Russ, G. et al. European Thyroid Association Guidelines for Ultrasound Malignancy Risk Stratification of Thyroid Nodules in Adults: The EU-TIRADS. Eur Thyroid J 6, 225-237, doi:10.1159/000478927 (2017).

5. Shin, J. H. et al. Ultrasonography Diagnosis and Imaging-Based Management of Thyroid Nodules: Revised Korean Society of Thyroid Radiology Consensus Statement and Recommendations. Korean J Radio/ 17, 370-395, doi:10.3348/kjr.2016.17.3.370 (2016).

6. Gharib, H. et al. American Association of Clinical Endocrinologists, American College of Endocrinology, and Associazione Medici Endocrinologi Medical Guidelines for Clinical Practice for the Diagnosis and Management of Thyroid Nodules-2016 Update. Endocr Pract 22, 622-639, doi:10.4158/EP161208.GL (2016).

7. Castellana, M. et al. Performance of Five Ultrasound Risk Stratification Systems in Selecting Thyroid Nodules for FNA. J Clin Endocrinol Metab 105, doi:10.1210/clinem/dgz170 (2020).

8. Kim, P. H. et al. Diagnostic Performance of Four Ultrasound Risk Stratification Systems: A Systematic Review and Meta-Analysis. Thyroid 30, 1159-1168, doi:10.1089/thy.2019.0812 (2020).

9. Ha, E. J., Na, D. G., Moon, W. J., Lee, Y. H. \& Choi, N. Diagnostic Performance of Ultrasound-Based Risk-Stratification Systems for Thyroid Nodules: Comparison of the 2015 American Thyroid Association Guidelines with the 2016 Korean Thyroid Association/Korean Society of Thyroid Radiology and 2017 American College of Radiology Guidelines. Thyroid 28, 1532-1537, doi:10.1089/thy.2018.0094 (2018).

10. Hoang, J. K. et al. Interobserver Variability of Sonographic Features Used in the American College of Radiology Thyroid Imaging Reporting and Data System. AJR Am J Roentgeno/ 211, 162-167, doi:10.2214/AJR.17.19192 (2018).

11. Fujioka, T. et al. Distinction between benign and malignant breast masses at breast ultrasound using deep learning method with convolutional neural network. Japanese Journal of Radiology 37, 466-472, doi:10.1007/s11604-019-00831-5 (2019).

12. Byra, M. et al. Breast mass classification in sonography with transfer learning using a deep convolutional neural network and color conversion. 46, 746-755, doi:https://doi.org/10.1002/mp.13361 (2019).

13. Becker, A. S. et al. Classification of breast cancer in ultrasound imaging using a generic deep learning analysis software: a pilot study. Br J Radio/ 91, 20170576, doi:10.1259/bjr.20170576 (2018).

14. Buda, M. et al. Management of Thyroid Nodules Seen on US Images: Deep Learning May Match Performance of Radiologists. Radiology 292, 695-701, doi:10.1148/radiol.2019181343 (2019).

15. Li, X. et al. Diagnosis of thyroid cancer using deep convolutional neural network models applied to sonographic images: a retrospective, multicohort, diagnostic study. Lancet Oncol 20, 193-201, doi:10.1016/S1470-2045(18)30762-9 (2019).

16. Zhao, W. J., Fu, L. R., Huang, Z. M., Zhu, J. Q. \& Ma, B. Y. Effectiveness evaluation of computer-aided diagnosis system for the diagnosis of thyroid nodules on ultrasound: A systematic review and meta-analysis. Medicine 98, e16379, doi:10.1097/MD.0000000000016379 (2019).

17. Koh, J. et al. Diagnosis of thyroid nodules on ultrasonography by a deep convolutional neural network. Sci Rep 10, 15245, doi:10.1038/s41598-020-72270-6 (2020).

18. Lee, J. H. et al. Sonographic and cytopathologic correlation of papillary thyroid carcinoma variants. J Ultrasound Med 34, 1-15, doi:10.7863/ultra.34.1.1 (2015).

19. Wang, L. et al. Automatic thyroid nodule recognition and diagnosis in ultrasound imaging with the YOLOv2 neural network. World J Surg Onco/ 17, 12, doi:10.1186/s12957-019-1558-z (2019).

20. Jeon, E. J. et al. Ultrasonographic Characteristics of the Follicular Variant Papillary Thyroid Cancer According to the Tumor Size. $J$ Korean Med Sci 31, 397-402, doi:10.3346/jkms.2016.31.3.397 (2016).

21. Kim, D. S. et al. Sonographic features of follicular variant papillary thyroid carcinomas in comparison with conventional papillary thyroid carcinomas. J Ultrasound Med 28, 1685-1692, doi:10.7863/jum.2009.28.12.1685 (2009).

22. Song, Y. S. et al. Ultrasonographic Differentiation Between Nodular Hyperplasia and Neoplastic Follicular-Patterned Lesions of the Thyroid Gland. Ultrasound Med Biol 42, 1816-1824, doi:10.1016/j.ultrasmedbio.2016.03.025 (2016).

23. Rago, T. et al. Combined clinical, thyroid ultrasound and cytological features help to predict thyroid malignancy in follicular and Hupsilonrthle cell thyroid lesions: results from a series of 505 consecutive patients. Clin Endocrinol (Oxf) 66, 13-20,

Page $10 / 13$ 
doi:10.1111/j.1365-2265.2006.02677.x (2007).

24. McHenry, C. R. \& Phitayakorn, R. Follicular adenoma and carcinoma of the thyroid gland. Oncologist 16, 585-593, doi:10.1634/theoncologist.2010-0405 (2011).

25. Cibas, E. S. \& Ali, S. Z. The 2017 Bethesda System for Reporting Thyroid Cytopathology. Thyroid 27, 1341-1346, doi:10.1089/thy.2017.0500 (2017).

\section{Figures}

Figure 1

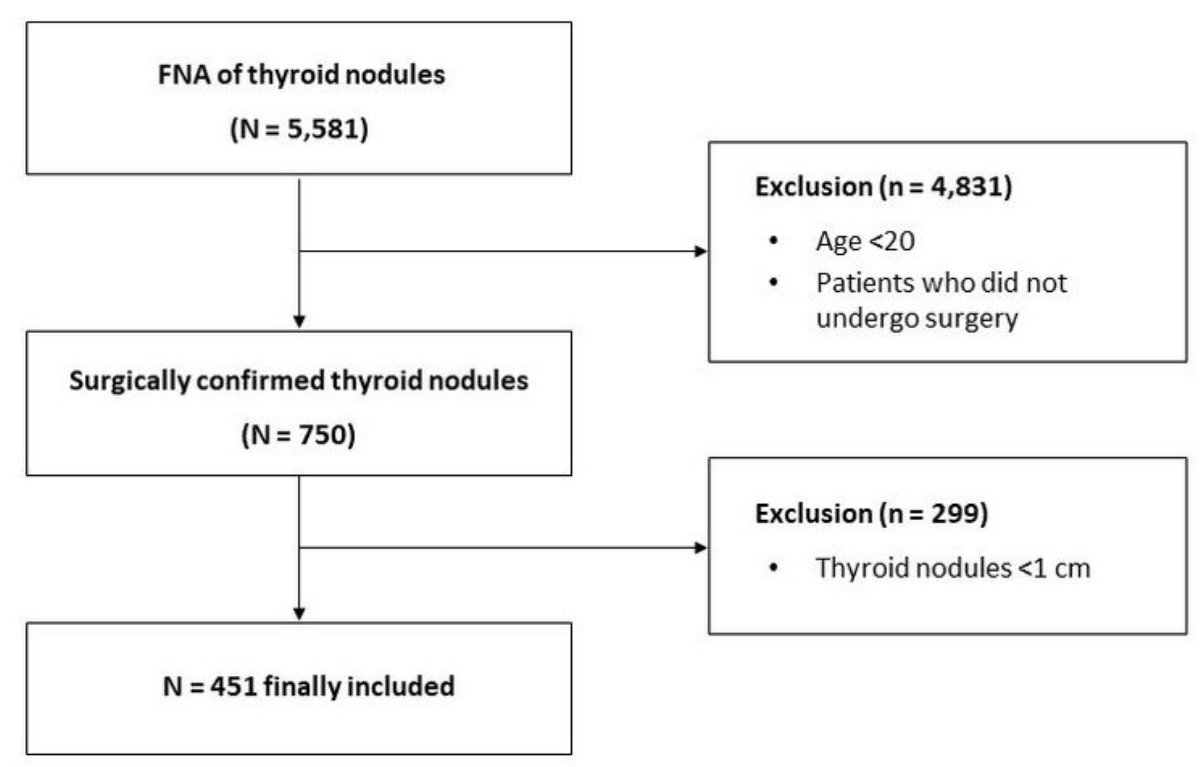

Figure 1

Flow diagram of study participants. FNA, fine-needle aspiration. 
Figure 2

A

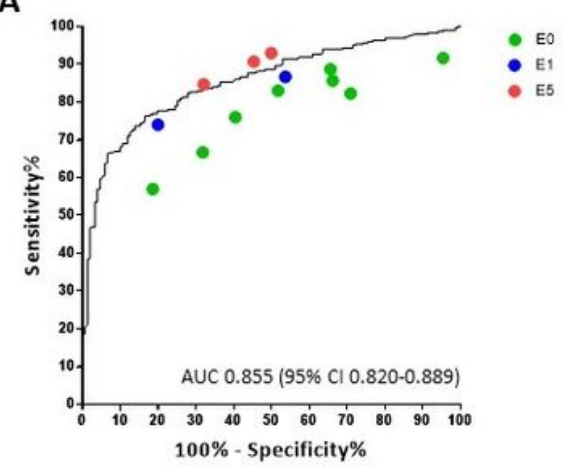

D

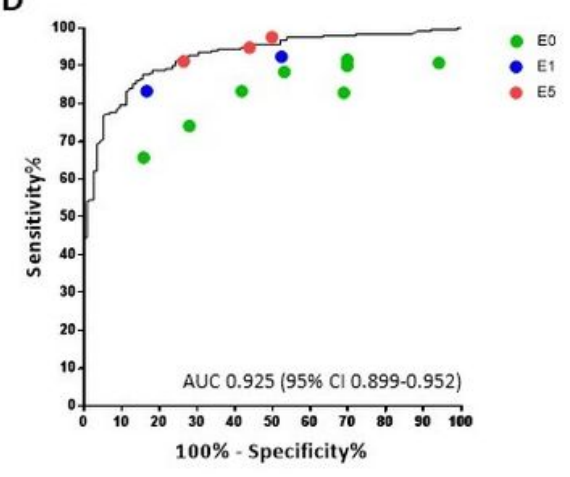

B

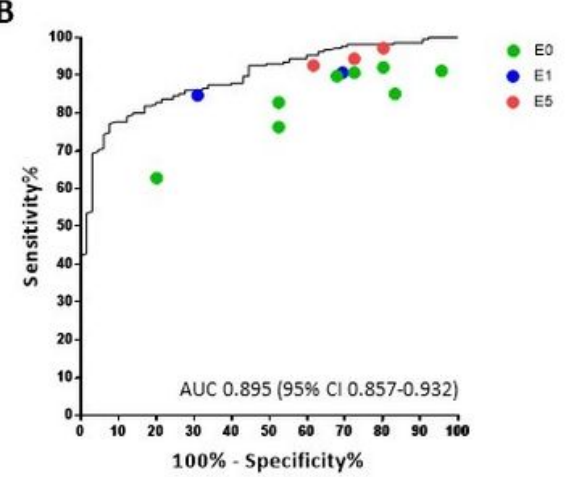

E

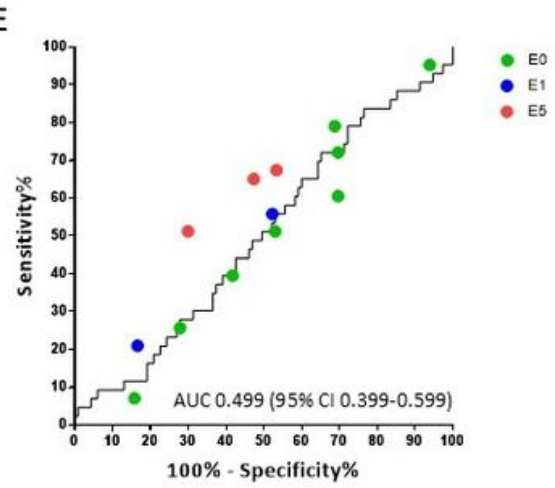

C

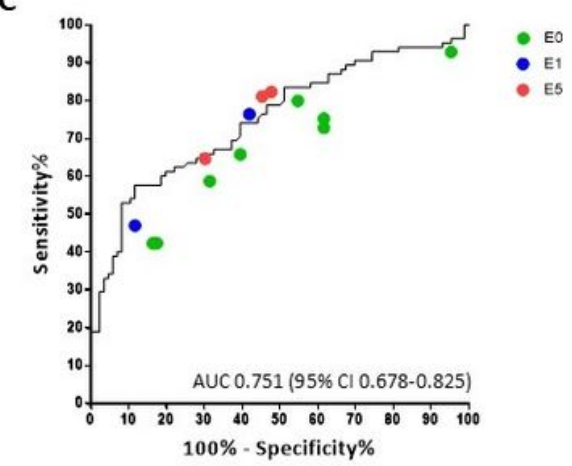

\section{Figure 2}

Comparison of diagnostic performance between CAD and physicians with different levels of experience The ROC curves and AUC of CAD in the diagnosis of thyroid nodules are demonstrated in black solid lines in each graph for (A) all nodules, (B) nodules with a size $<2 \mathrm{~cm}$, (C) nodules with a size $\geq 2 \mathrm{~cm}$, (D) nodules diagnosed as CPTC, and (E) nodules diagnosed as FTC and fvPTC. Dots on each graph indicate the diagnostic performance (sensitivity and specificity) of the individual physicians in the E0 (green), E1 (blue), and E5 (red) groups. CAD, computer-aided diagnosis; ROC, receiver operating characteristic curve; AUC, area under the curve; CPTC, conventional papillary thyroid carcinoma; FTC, follicular thyroid carcinoma; fvPTC, follicular variant papillary thyroid carcinoma; E0, E1, E5, physicians with $0,1,5$ years of experience, respectively.

\section{Figure 3}

A

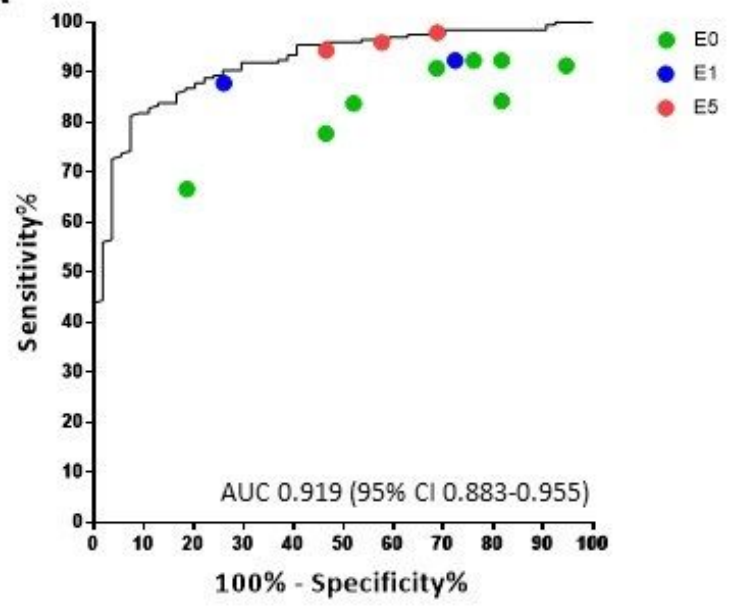

B

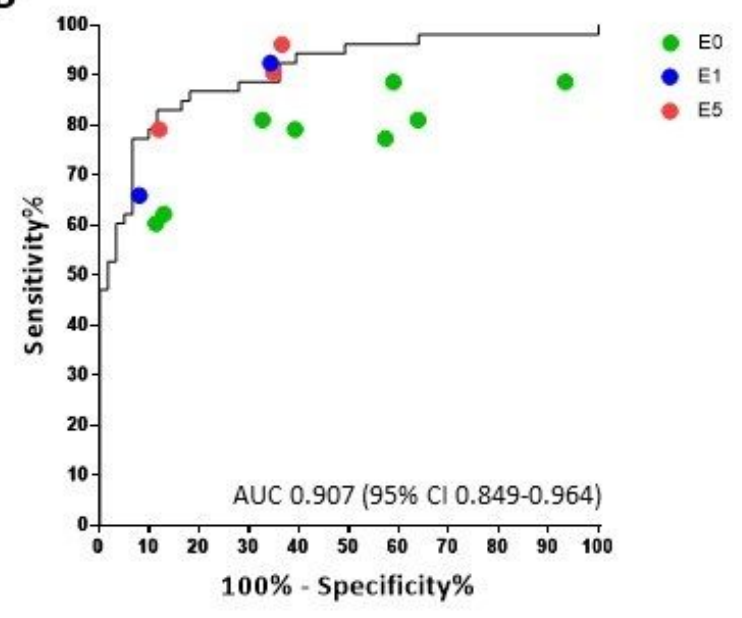

Figure 3 
Diagnostic performance of CAD and physicians for CPTC according to nodule size The ROC curves and AUC of CAD for CPTC are presented for thyroid nodule with $(A)$ a size $<2 \mathrm{~cm}$, and $(B)$ a size $\geq 2 \mathrm{~cm}$. Dots on each graph indicate the diagnostic performance (sensitivity and specificity) of the individual physicians in the E0 (green), E1 (blue), and E5 (red) groups. CAD, computer-aided diagnosis; ROC, receiver operating characteristic curve; AUC, area under the curve; CPTC, conventional papillary thyroid carcinoma.

\section{Supplementary Files}

This is a list of supplementary files associated with this preprint. Click to download.

- AlthyroidSupplementaryTable20210515.docx 